\title{
Lyotropic Mesomorphisms of a Lamellar Liquid Crystalline Phase in Non-hydrous Condition: A Phospholipid Hydrated by Different Polar Solvents
}

\author{
Dong Kyu Lee ${ }^{\dagger}$ and Kwan Young Jeong* \\ Skin research institute, Korea Kolmar Corporation, 170-7, Seojeong-Ri, Jeonui-Myun, Yeongi-Gun, Chung-Nam, Korea \\ *E-mail: jeong@kolmar.co.kr \\ ${ }^{\dagger}$ Department of Engineering Chemistry, Chung-buk University, Chung-buk 361-783, Korea \\ Received November 7, 2009, Accepted February 25, 2010
}

\begin{abstract}
The lyotropic mesomorphism of lamellar liquid crystalline phase was examined by observing the swelling behavior of Distearoylphosphatidylcholine(DSPC) in glycerin and panthenol without water. The lyotropic mesomorphism was examined by using DSC, XRDs and Cryo-SEM. Increase of two polar solvents under non-hydrous condition showed distinctive differences in the lyotropic mesomorphism from forming different anisotropic structures with DSPC. Glycerin did not affect to the crystalline region of lamellar phase, whereas typical swelling mesomorphism was shown in the noncrystalline region. In contrast, panthenol showed some effect on the crystalline region, but common swelling mesomorphism was found in the non-crystalline region. In this case, the isopropyl and propyl groups in panthenol were the main factor to affect to the lipophilic domain in the crystalline region of lamellar phase. Also, it was found that the formation of well-arranged lamellar structure only by introducing glycerin and panthenol as a solvent without water, was possible. These results were confirmed by examination of the swelling mesomorphism of liquid crystal membrane triggered by introducing the two polar solvents.
\end{abstract}

Key Words: Lyotropic mesomorphism, Lamellar, Non-hydrous, Repeat distance, Swelling

\section{Introduction}

The structure of stratum corneum is mainly composed of crystalline lamellar structure densely packed with dead cells containing keratin, which is identical to brick and mortar-like structure. ${ }^{1}$ Most active ingredients applied to the skin are diffused through the crystalline lamellar structures among cells. This densely packed structure disturbs the diffusion of active ingredients and lowers the efficiency of drug delivery, thereby decreasing the effectiveness of whitening, anti-aging and moisturizing effect. ${ }^{2}$ In contrast, such structure protects inner organs from external environment and acts as the main factor to maintain the homeostasis of human skin.

In terms of drug delivery, the penetration rate of drugs into human skin can be enhanced by the combination of polar solvents with low molecular weights and phospholipids which could effectively decrease the barrier function of stratum corneum. ${ }^{3}$ In pharmacology, many studies have performed transdermal assessment on testosterone, haloperidol, flurbiprofe and other various active ingredients by using propylene glycol (PG), polyethylene glycol (PEG), their derivatives, ethyl alcohol and glycerol monooleate as penetrating enhancers. ${ }^{4-6}$ However there were limits in designing liquid crystal membranes with similar structure to stratum corneum by the combination of phospholipids and polar solvents.

The lyotropic mesomorphism of lamellar crystalline phase is generated by using solvents on amphoteric lipids which are solid or liquid. Among various solvents, the most important substance to combine with amphoteric lipids is water. ${ }^{7}$ Owing to new calorimetric methods, the energetics of the binding of water molecules to model membranes has been determined. ${ }^{8,9}$ Several related studies have mentioned of lyotropic mesomorphism under non-hydrous conditions but was limited to organic solvents and macromolecular systems. ${ }^{10}$

Phospholipids are one of the typical amphoteric lipids and when dispersed in water, they can form various types of phases dependent on their molecular structures and dispersion conditions. Those forms are composed of hydrated phospholipid aggregates and these aggregation processes including the phase transition is caused by the hydrophobic interaction of the aggregates. These phase transitions are affected by the lipid concentration and temperature. Those occur from the two mechanisms as the following; 1) Chain melting transition caused by increasing the configurational entropy of lipid chains. 2) Nonlamellar phase transition caused by the instant curvature tendency of bilayer phase. Further details of phase transition were discussed in Cevc and Marsh. ${ }^{11-12}$

As seen from the above, most of researches for the behaviors of LC formation have been conducted in the region of the mixtures of phospholipids and water. However, many anti-aging ingredients such as retinol, lipophillic derivatives of vitamin c and tocopherol have been limited in the broad application of cosmetic and pharmaceutical fields, because of their oxidative characteristics which become unstable at the moment of facing with water molecules. Therefore, we did more detailed studies about the swelling behaviors between DSPC and other solvents (glycerin and panthenol) under the non-existing condition of water, so as to protect from these oxidative damages and develop cosmetics and pharmaceutics having superior anti-aging effects. In this study, glycerin and panthenol, which have 3 hydroxyl groups but different hydrophilic character, were chosen as the main solvents swelling phospholipids, packing lipids with stratum corneum and enhancing skin moisturization. The lyotropic mesomorphism of liquid crystalline lamellar phase formed by 
DSPC and two solvents were examined with DSC, XRDs and Cryo-SEM. Additionally, a new area of lyotropic mesomorphism was discovered in terms of the possibility to apply to recover damaged skin barrier and cure atopy symptoms dermatologically.

\section{Materials and Methods}

Materials. DSPC was purchased from Lipoid(Germany). The two solvents, Glycerin(Gly) and D-Panthenol(Pan) were purchased from Merck(Germany). The solvents are all cosmeticgrade without any pre-treatment.

Methods.

Sample preparation: DSPC was mixed with Gly and Pan. The mixture was then heated up to $80{ }^{\circ} \mathrm{C}$ to be completely dissolved. The solution was cooled to around $60{ }^{\circ} \mathrm{C}$. And then, it was cooled slowly again to $35^{\circ} \mathrm{C}$. After completion, it was kept around $10{ }^{\circ} \mathrm{C}$ for 3 days prior to analysis.

DSC analysis: Thermotropic mesomorphism was examined by using TA instrument (TA4100 model) at $20^{\circ} \mathrm{C} \sim 120^{\circ} \mathrm{C}$ with the heating rate of $2^{\circ} \mathrm{C} / \mathrm{min}$. The volume of each sample was approximately $10 \mathrm{mg}$ and was sealed with aluminum sample cell. Mesomorphism was performed under nitrogen gas and phase transition temperature $\left(T_{c}\right)$ and enthalpy change $(\Delta H)$ were observed to examine the formation rate of liquid crystalline phase and the phase transition depending on temperature.

XRD analysis: XRD spectras were analyzed with XDS 2000 model (SCINTAG INC., USA) at $24{ }^{\circ} \mathrm{C}$. As seen in Fig. 1 , XRD experiments were carried out with $\mathrm{Ni}$-filtered $\mathrm{Cu} \mathrm{K} \alpha$-ray $(\lambda=$ $1.54 \AA$ ) using photo detection.

SAXS data was recorded by using a position sensitive proportional counter (multichannel analyzer, SCINTAG INC., USA) with camera size of $350 \mathrm{~mm}$. Diffraction patterns are plotted as an equation of Q. Q is regarded as a scattering vector and calculated from equation $Q=4 \pi(\sin \theta) / \gamma$. D is the repeating distance of lamellar structure and $\mathrm{n}$ is the degree of diffraction peak. Repeating distance of lamellar structure is calculated from the equation, Qn $=2 \pi \mathrm{n} / \mathrm{D}$ followed by J. Zbytovska et al. ${ }^{13}$

WAXS data was recorded by a high-sensitive X-ray film (66.0mm, Fuji Medical X-ray Film) and a flat-plate film cassette. The samples were sealed inside a thin-walled glass capillary tube (outer diameter $1.0 \mathrm{~mm}$ ). The sealed samples were then measured on stable holders $\left( \pm 0.2^{\circ} \mathrm{C}\right)$. The interlayer distance between bilayers can be calculated from the following equation (1) $: \mathrm{d}=$ interlayer distance, $\lambda=$ wave length of X-ray $(1.54 \AA)$,

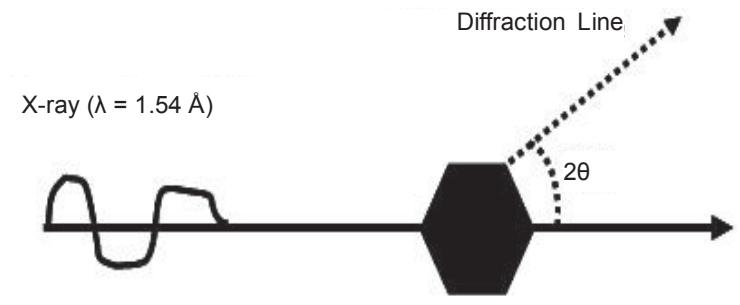

Figure 1. Representitive scheme of $X$-ray diffraction; $\lambda$ is the wave length of X-ray and $2 \theta$ is the diffraction angle to determine the crystalline structure and its arrangement.
$2 \theta=$ Bragg's angle

$$
\mathrm{D}=\lambda /(2 \sin \theta)
$$

Cryogenic SEM (Scanning Electron Microscope) analysis : The structure of crystalline lamellar phase was examined by Cryo-SEM(HITACHI S-4700 Model). The sample was placed on Specimen Carrier $(4.5 \mathrm{~mm} \times 3 \mathrm{~mm}$ ) to make sandwich form and was instantly freezed at $-180{ }^{\circ} \mathrm{C}$ by using Jet Freezer Device (JFD 030, Switzerland BAL-TEC). The sample was then inserted into a chamber maintaining the temperature of $-120{ }^{\circ} \mathrm{C}$ by using High Vacuum Cryo transfer System (VCT 100, Switzerland BAL-TEC). Inside the chamber, freeze fracture process to cut one end of sandwich form sample is performed and followed by etching for $5 \mathrm{~min}$ at $-100{ }^{\circ} \mathrm{C} \sim 110{ }^{\circ} \mathrm{C}$. The sample was then coated with Platinum and was inserted into Cryo-SEM for observation.

\section{Results and Discussion}

Lyotropic interaction of Gly and Pan with DSPC from SAXS. SAXS has been widely used for the analysis of amphoteric lipid structures and their lamellar liquid crystalline phase structures in polar medias. ${ }^{14-16}$ It is known that phospholipids form lamellar structures comprising of multi-bilayers having several nano meters of repeat distance under few types of polar solvents such as glycols. Such structures stimulate Bragg diffraction.

As shown in Fig. 2, the repeat distance (D) is shown as the sum of lipid bilayer and interlayer thickness. The thickness of interlayer is expressed as d. These data all correspond to SAXS and WAXS analysis results. Non-tilted bilayer distance could be theoretically calculated as $55.08 \AA$ : $(18 \times 1.53 \AA) \times 2,18$ is the number of hydrocarbon, $1.53 \AA$ is the length of methylene group $\left(-\mathrm{CH}_{2}\right), 2$ stands for bilayer. ${ }^{17}$

$\mathrm{Oh}$ and Jeong reported the schematic representation of lamellar crystalline phases complexed between DSPC and propylene glycol. $^{18}$

The introduction of solvents into lipid structures increases the expansion of polar area(c), leading to structure expansion. The SAXS patterns of DSPC tended to be shifted left-side by

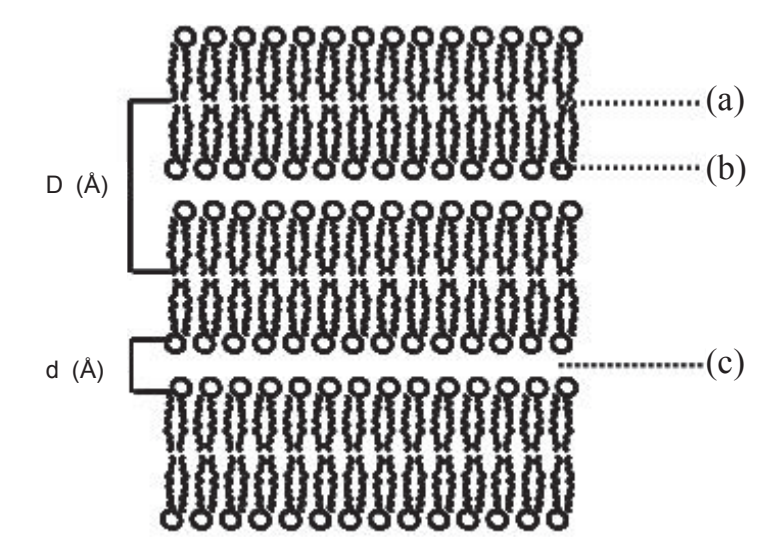

Figure 2. Schematic representation of the lamellar structure being composed of DSPC when complexed with a polar solvent. (a), (b) and (c) correspond to the regions affected by solvents. 
(A)

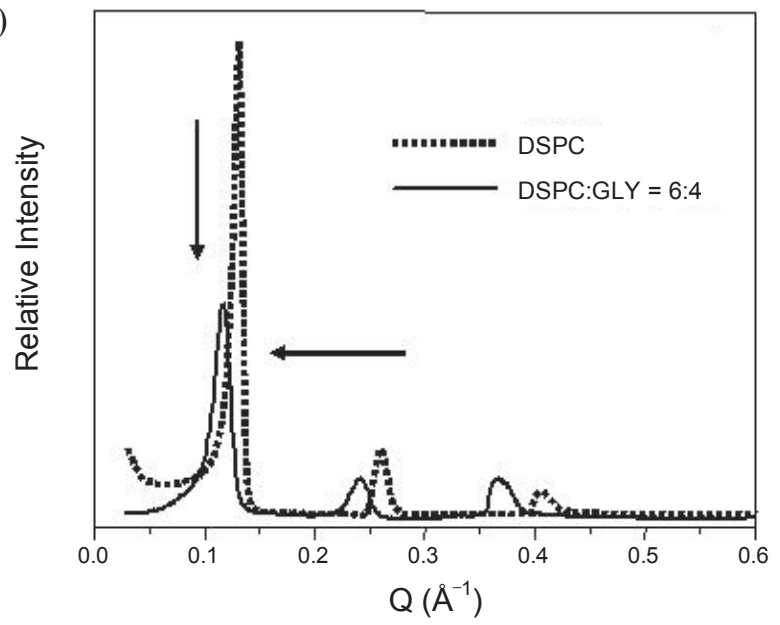

(B)

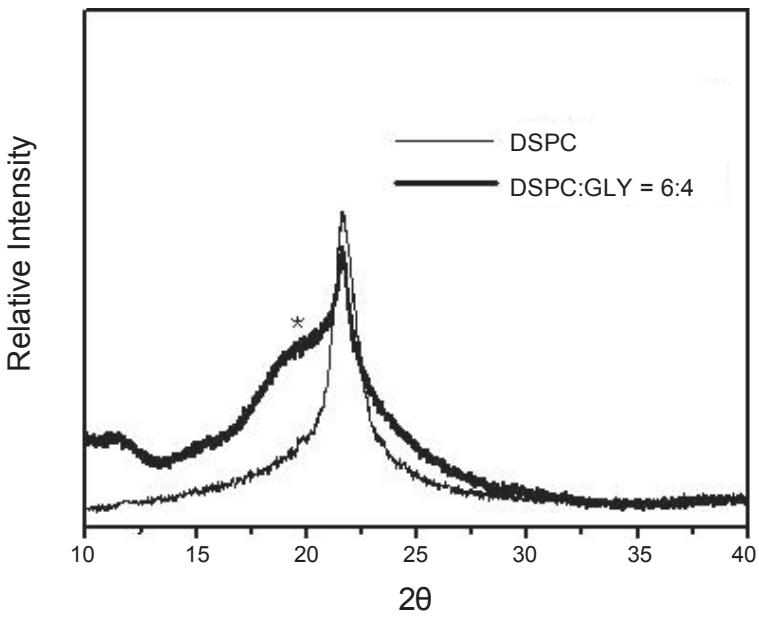

Figure 3. SAXS(A) and WAXS(B) explanation of the swelling mesomorphism of lipid matrix by incorporating Gly as a solvent.

increased bilayer thickness under the introduction of solvent (Fig. 3(A)). WAXS patterns showed broad overlapping patterns to the left region of the main peak (Fig. 3(B)).

As shown in Fig. 3(A), SAXS patterns showed three separated peaks when GLY is introduced into DSPC, which were similar to DSPC itself.

The 1st, 2nd, and 3rd peaks in all SAXS patterns were expressed as $2 \mathrm{nQn}(\mathrm{n}=$ number) and these all confirmed the formation of lamellar crystalline structures and the non-existence of any separated phase. SAXS pattern for DSPC itself without GLY showed values of $q=0.134,264,405 \AA^{-1}$. The lamellar repeat distance (D) showed $47.01 \AA^{-1}$, which was slightly shorter than the theoretical estimate. As shown in Fig. 4, SAXS patterns showed the left-side shifting behaviors depending on the GLY content to be incorporated into DSPC lamellar structure. Eventually, the increase of lamellar repeat distance(D) occurred.

The $\mathrm{D}$ values were plotted as a function of GLY/DSPC ratio. $D$ values showed a linear- increasing pattern from $50.18 \AA$ to $58.01 \AA$. It suggests that GLY rarely affects to the crystalline region and is being swollen only in the non-crystalline region due to its relatively low lipophilicity from 3 methylene groups, in comparison to its high hydrophilicity derived from three hydroxyl groups. Similar to the decrease of $\Delta \mathrm{H}$ in DSC patterns,

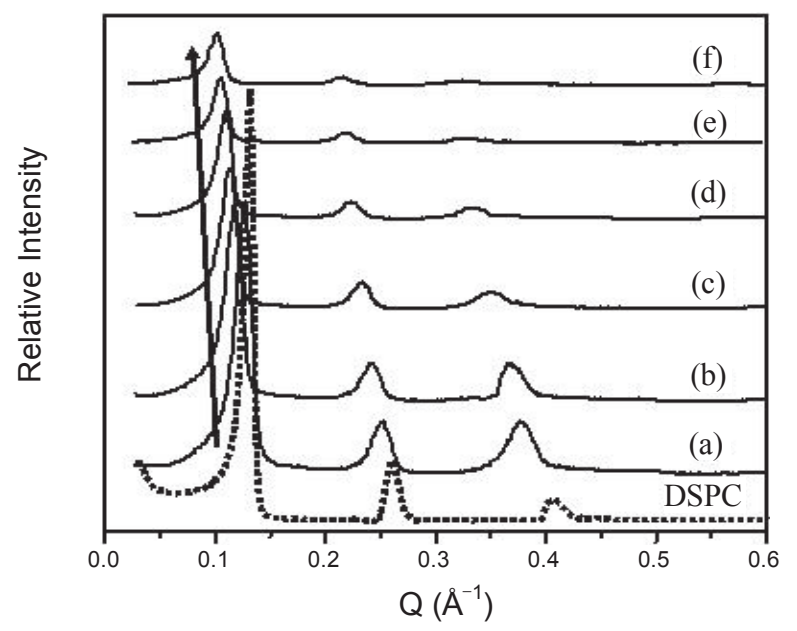

Figure 4. SAXS patterns of crystalline domains in the lamellar structure of DSPC depending on the amount of GLY : DSPC, a) $25.0 \% \mathrm{w} / \mathrm{w}$ GLY, b) $40.0 \% \mathrm{w} / \mathrm{w}$ GLY, c) $55.0 \% \mathrm{w} / \mathrm{w}$ GLY, d) $70.0 \% \mathrm{w} / \mathrm{w}$ GLY, e) $80.0 \%$ w/w GLY, f) $90.0 \%$ w/w GLY.

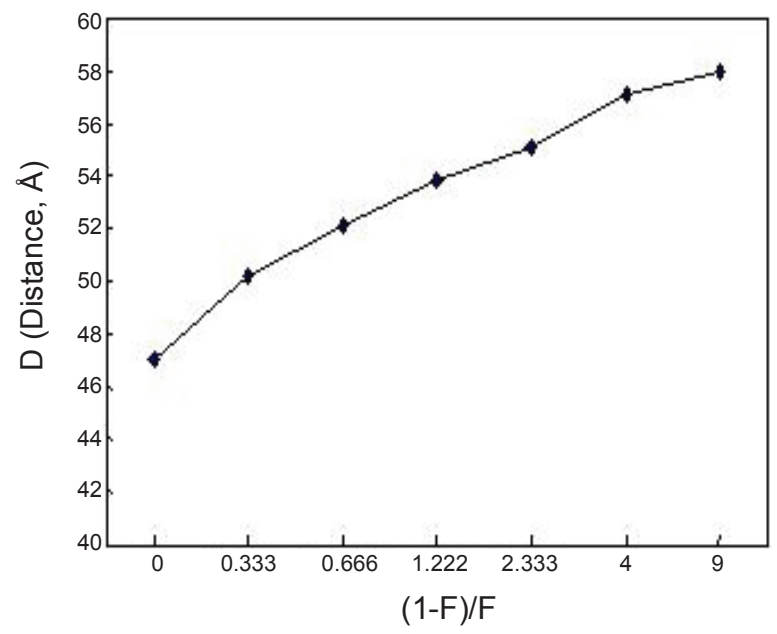

Figure 5. Variances of the repeat distance(D) in the mixtures of DSPC and GLY at $24{ }^{\circ} \mathrm{C} v s$. the ratio GLY/DSPC, F is the weight fraction of DSPC.

the intensity of the 1st order peak decreased depending on GLY content, and this result is meant to be the reduction of the crystalline region in the lamellar structure.

According to G. Klose et al., the D value increased in linear form from $50 \AA$ to $63 \AA$ when water is introduced into egg phosphatidylcholin membrane. ${ }^{19}$ T. de Vringer et al. also stated that $D$ values increased from $70 \AA$ to $89 \AA$ when water was introduced into PGM(Polyglycerylmyristate). ${ }^{20}$ Therefore, when polar solvents other than water such as GLY are introduced into DSPC lamellar structure, they interact with (b) region shown in Fig. 2 and are swollen into (c) region. The swelling degree was smaller than water molecules but the swelling itself was clearly visible.

In the case of introducing PAN, all SAXS patterns were expressed by $2 \mathrm{nQn}(\mathrm{n}=$ number) in the 1st, 2nd, 3rd peaks and these all formed lamellar crystalline structures. The existence of a separated phase which shows longer distance compared to the 


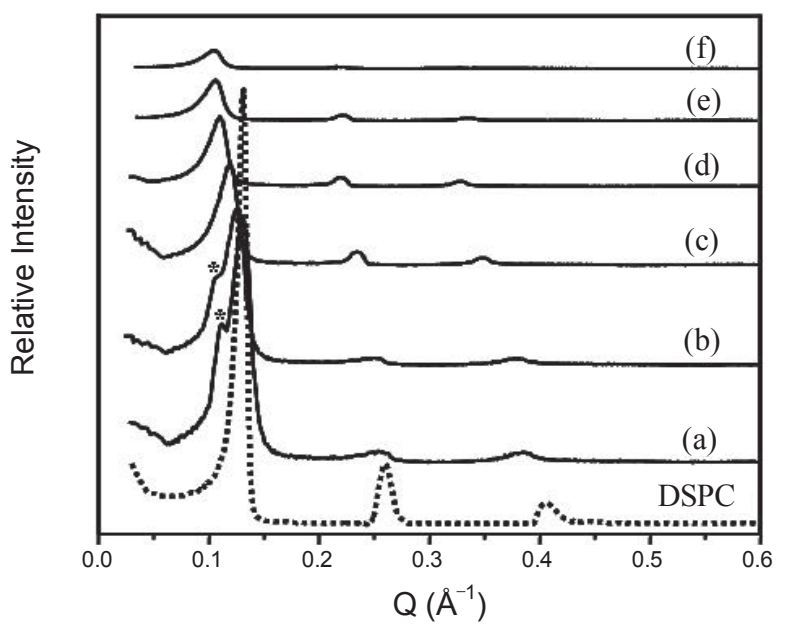

Figure 6. SAXS patterns of crystalline domains in the lamellar structure of DSPC depending on the amount of PAN : DSPC, a) $25.0 \% \mathrm{w} / \mathrm{w}$ PAN, b) $40.0 \% \mathrm{w} / \mathrm{w}$ PAN, c) $55.0 \% \mathrm{w} / \mathrm{w}$ PAN, d) $70.0 \% \mathrm{w} / \mathrm{w}$ PAN, e) $80.0 \%$ $\mathrm{w} / \mathrm{w}$ PAN, f) $90.0 \% \mathrm{w} / \mathrm{w}$ PAN.

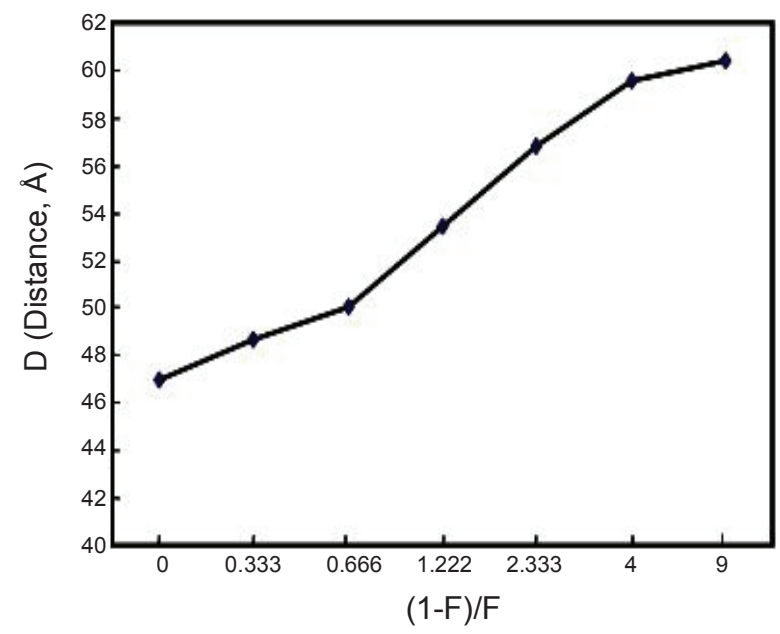

Figure 7. Variances of the repeat distance(D) in the mixtures of DSPC and PAN at $24^{\circ} \mathrm{C} v s$. the ratio PAN/DSPC, F is the weight fraction of DSPC.

main crystalline peak was found up to $40 \%$ of PAN content. But in concentrations of over $40 \%$ PAN, all data showed the tendency to be left-side shifted, depending on the PAN content and the increase of lamellar repeat distance (D) similar to those of GLY. The D values were plotted as a function of PAN/DSPC ratio (Fig. 7). D values showed the linear-increasing patterns from $48.64 \AA$ to $60.41 \AA$. Such pattern correlated with increasing PAN concentration. It showed different swelling behaviors below $40 \%$ PAN in comparison to over $55 \%$ PAN, but its overall swelling behavior was comparatively well-progressed.

In addition, the distortion of DSPC hydrocarbon chain in the lamellar crystalline phase occurred from the weak lipophilic interaction of isobutylene and propylene groups in PAN. This distortion affects to the crystalline region and makes the lamellar repeat distance (D) comparatively longer than those of GLY. Similar to GLY, the intensity of the 1st order peak decreased de-

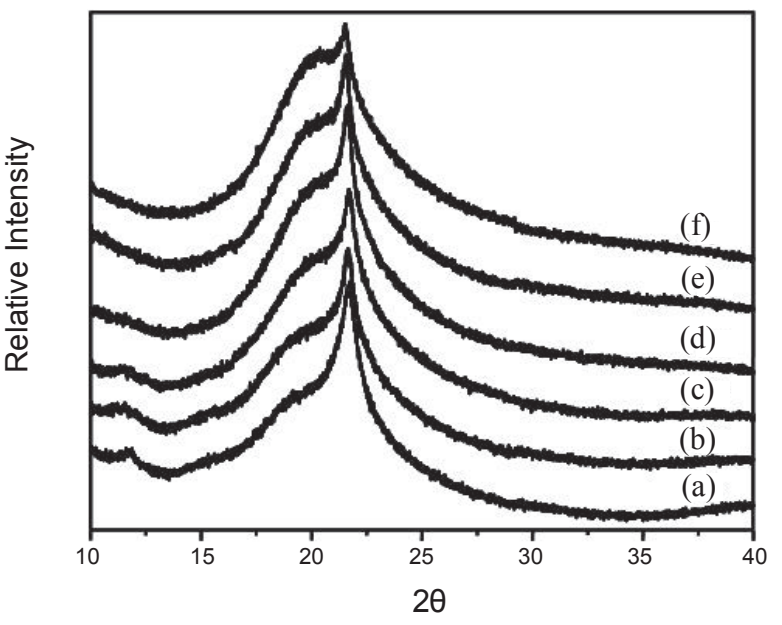

Figure 8. WAXS patterns of non-crystalline domains in the lamellar structure of DSPC depending on the amount of GLY : a) $25.0 \% \mathrm{w} / \mathrm{w}$ GLY, b) $40.0 \% \mathrm{w} / \mathrm{w}$ GLY, c) $55.0 \% \mathrm{w} / \mathrm{w}$ GLY, d) $70.0 \% \mathrm{w} / \mathrm{w}$ GLY, e) $80.0 \% \mathrm{w} / \mathrm{w}$ GLY, f) $90.0 \% \mathrm{w} / \mathrm{w}$ GLY.

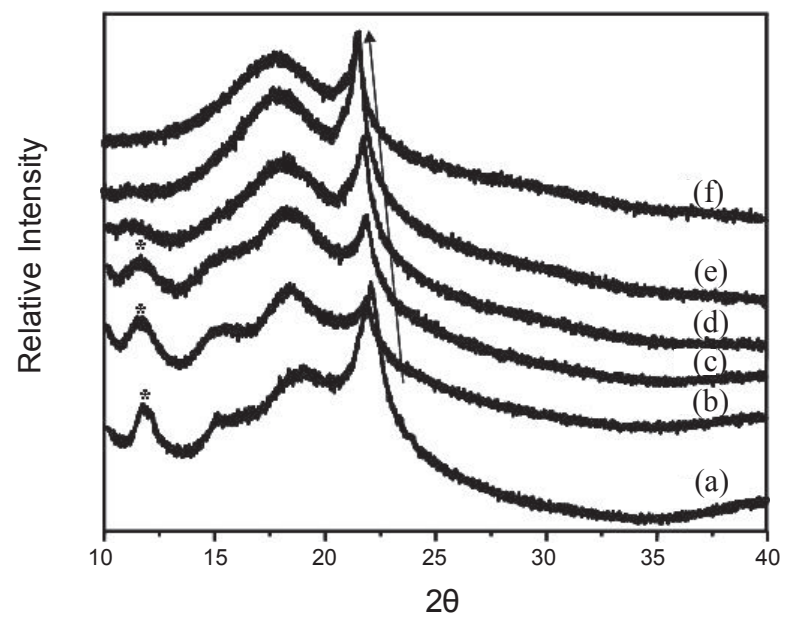

Figure 9. WAXS patterns of non-crystalline domains in the lamellar structure of DSPC depending on the amount of PAN : a) $25.0 \% \mathrm{w} / \mathrm{w}$ PAN, b) $40.0 \% \mathrm{w} / \mathrm{w}$ PAN, c) $55.0 \% \mathrm{w} / \mathrm{w}$ PAN, d) $70.0 \% \mathrm{w} / \mathrm{w}$ PAN, e) $80.0 \% \mathrm{w} / \mathrm{w}$ PAN, f) $90.0 \% \mathrm{w} / \mathrm{w}$ PAN.

pendently on PAN content, corresponding to the decrease of $\Delta \mathrm{H}$ in DSC patterns. Therefore, when PAN is introduced into the DSPC lamellar structure, it was found that the polar interaction would occur in (b) region shown in Fig. 2 and PAN would give some effect to the region between (a) and (b) due to its lipophilicity as well as being swollen in the (c) region.

Lyotropic interaction of Gly and Pan with DSPC from WAXS. WAXS patterns showed the interaction between electron dense phosphate parts in DSPC head groups and polar solvents. Examining these patterns enable calculation of the interlayer distance (d) between (b) and (c) regions.

All samples showed slightly diffused or broad peaks around the main peak $\left(2 \theta=19.5^{\circ} \pm 0.4\right)$ and the peak intensity increased dependently on the GLY content. The diffused peaks occurred as a result of electron density difference between lipid bilayers and solvent molecules. It can be concluded that the head groups of 
(A)
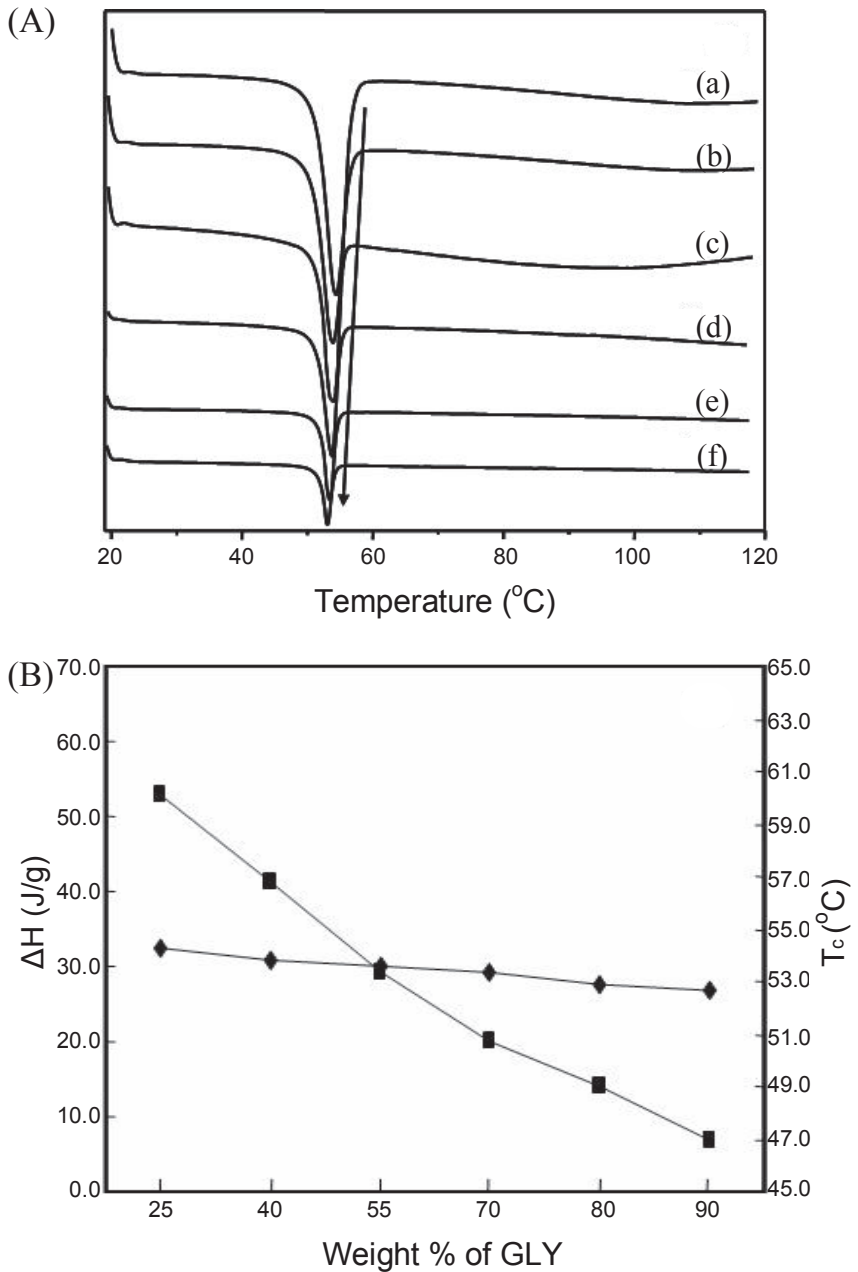

Figure 10. DSC patterns (A) of DSPC lamellar phase swellon by GLY during heating process from $20^{\circ} \mathrm{C}$ to $120^{\circ} \mathrm{C}$ and their comparisions (B) depending on the GLY content: a) $25.0 \% \mathrm{w} / \mathrm{w}$ GLY, b) $40.0 \% \mathrm{w} / \mathrm{w}$ GLY, c) $55.0 \% \mathrm{w} / \mathrm{w}$ GLY, d) $70.0 \% \mathrm{w} / \mathrm{w}$ GLY, e) $80.0 \% \mathrm{w} / \mathrm{w}$ GLY, f) $90.0 \% \mathrm{w} / \mathrm{w}$ GLY.

DSPC and GLY together formed a complex. ${ }^{21}$ Also, one sharp and other diffused peaks show miscibility between DSPC and polar solvents under solvent concentrations at which separated interlayers may form. DSPC under polar solvents may have a limited solubility according to their miscibility. ${ }^{22}$ Fig. 8 shows changes in WAXS patterns according to the content of GLY. The interlayer distance(d) of the lamellar phase which is swelled by GLY can be calculated from the main diffraction peak. Overall data from the above equation (1) show the interlayer distance (d) of $4.12 \AA \pm 0.02$ at $2 \theta=21.5^{\circ} \pm 0.1$. There were no correlations between the interlayer distance(d) and GLY content in this range. However, the intensity and area of the broad peak at $2 \theta=$ $19.5^{\circ} \pm 0.4$ increased with the GLY content. At $90 \%$ of GLY, the formation of almost one diffused peak was observed. This shows that GLY molecules interact with the hydrophilic interlayer in DSPC lamellar phase and form a complex structure. In other words, the interlayer located between lipid bilayers have been swollen by continuous insertion of GLY. These are the reason why GLY incoporated in DSPC lamellar phase is located at (c) region in Fig. 2 due to 3 hydroxyl groups. This layer is defined as
(A)
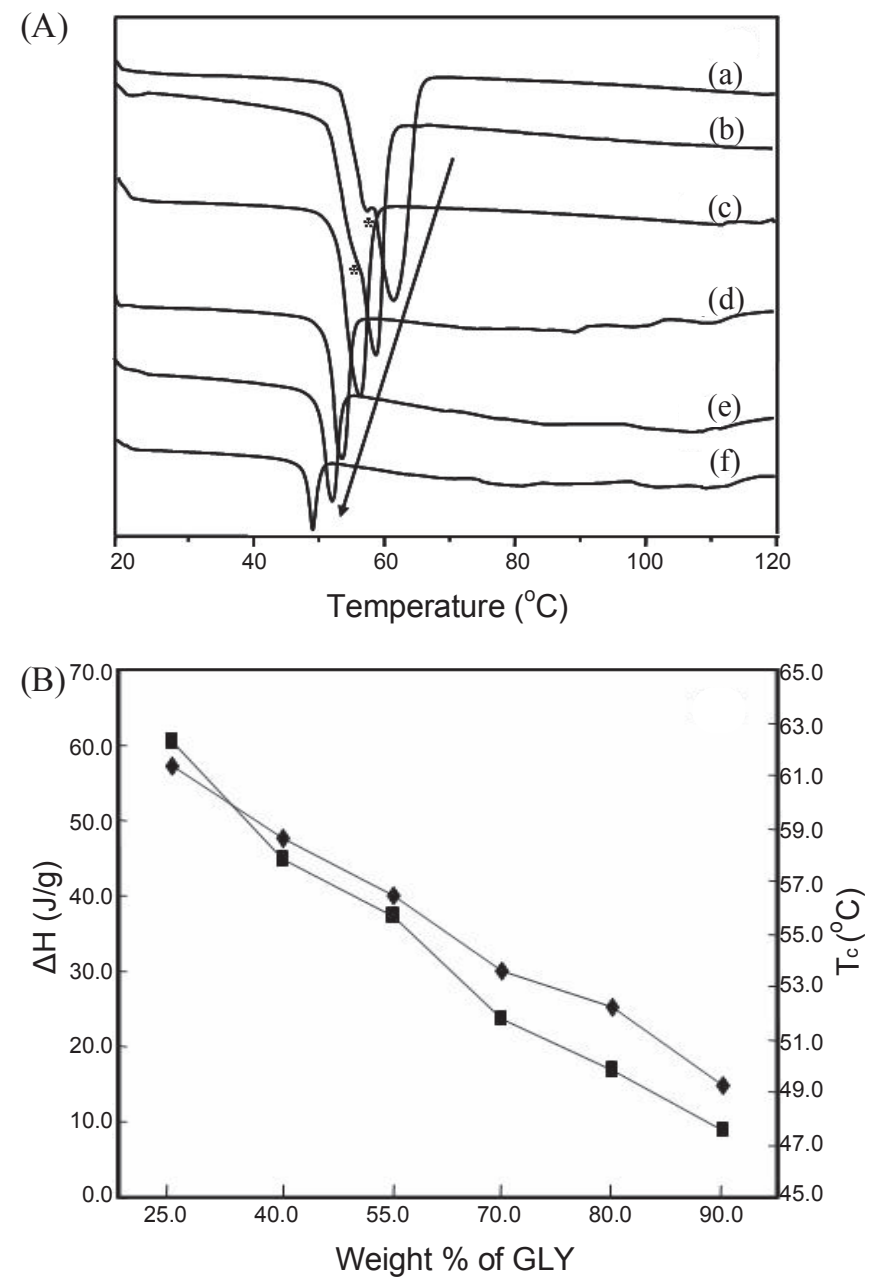

Figure 11. DSC patterns (A) of DSPC lamellar phase swellon by PAN during heating process from $20^{\circ} \mathrm{C}$ to $120^{\circ} \mathrm{C}$ and their comparisions (B) depending on the PAN content: a) $25.0 \% \mathrm{w} / \mathrm{w}$ PAN, b) $40.0 \% \mathrm{w} / \mathrm{w}$ PAN, c) $55.0 \% \mathrm{w} / \mathrm{w}$ PAN, d) $70.0 \% \mathrm{w} / \mathrm{w}$ PAN, e) $80.0 \% \mathrm{w} / \mathrm{w}$ PAN, f) $90.0 \%$ w/w PAN.

the interlayer between lipid bilayers and the polar groups of GLY penetrate into this region, eventually increasing electrostatic repulsion with the polar groups of DSPC. Such repulsion leads to the generation of non-crystalline phase having a longer interlayer distance and may cause the expansion of this region. However, polar groups in GLY have weaker interacting capabilities in comparison to water. Therefore, these peaks were expressed as diffused peaks and the interlayer distance also increased less than water.

Fig. 9 show changes in WAXS patterns according to the content of PAN. Up to $25 \%$ PAN, the main peak corresponding to the interlayer swollen by PAN was observed at $2 \theta=21.5^{\circ} \pm 0.1$ and it showed the tendency to be left-side shifted, depending on the increase of PAN content. It suggests that when PAN is being swollen in the lamellar matrix of DSPC, weak lipophilic groups exist in PAN molecule and slightly affect to the (a) and (b) region as shown in Fig. 2. This result is correlated with the previous result from SAXS. The swollen rate of PAN penetrating into the DSPC lamellar phase was shown irregular up to $55 \%$ of PAN and the formation of another phase was observed at around $2 \theta=$ 


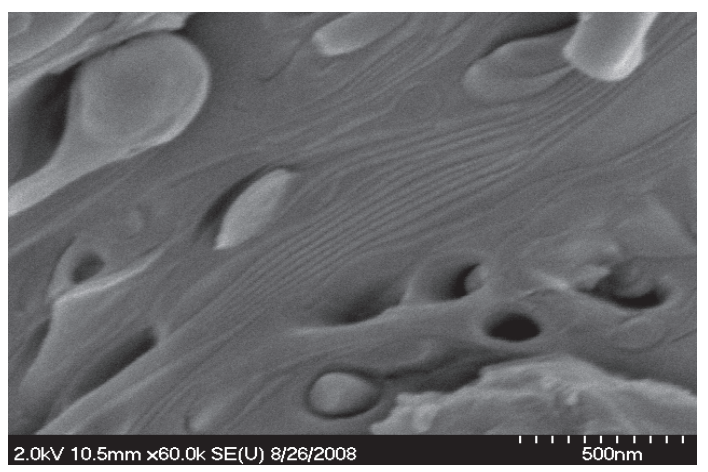

Figure 12. Cryo-SEM photographs of samples having the lamellar structure to be well-formed and well-swollen by GLY at 70.0\% w/w.

$11.6^{\circ} \pm 0.05$. In the case of PAN concentrations over $70 \%$, another phase disappeared and only diffused peak beside the main peak by the electron density contrast was observed. This is the result to correspond to the eutectic behavior from DSC patterns shown in Fig. 11. PAN, which has more lipophilic property than GLY, affects to the hydrocarbon located closely to the polar region in these lamellar phases and leads to the left-side shift of the main peak. It was found that the swelling behaviors in low levels of PAN don't work well to form another.

Thermotropic interaction of Gly and Pan with DSPC from DSC. Fig. 10 and Fig. 11 show the thermal mesomorphism of complex mixtures which are formed by the introduction of GLY and PAN into DSPC lamellar structure. Transition temperature $\left(\mathrm{T}_{\mathrm{c}}\right)$ means the point to change from $\alpha$-lamella state to isotropic state closely related to the melting temperature of diacyl groups in DSPC. As seen in Fig. 10(A), $\mathrm{T}_{\mathrm{c}}$ of the main transition peak decreased very slightly from $54.22^{\circ} \mathrm{C}$ to $52.22^{\circ} \mathrm{C}$ with the monotectic pattern. However, $\Delta \mathrm{H}$ showed almost linear-decreasing pattern as seen in Fig. 10(B), and the decreasing degree at this time decreased remarkably from $52.96 \mathrm{~J} / \mathrm{g}$ to $6.96 \mathrm{~J} / \mathrm{g}$. Similar to the XRD patterns mentioned above, GLY molecules did not show any effect to the crystalline region and is swollen only in the non-crystalline region due to its weak lipophilicity. In addition, although the formation area of liquid crystalline phase decreased dependent on GLY content, GLY was found to be wellswollen up to $90 \%$.

Fig. 11 shows the thermotropic behavior of DSPC lamellar phase in the case of incorporating PAN. Below $40 \%$ PAN, it was shown to be weak eutectic patterns as seen in Fig. 11(A) and it suggested that another phase is formed in this region.

The results were identical as well from XRDs. Tc of the main transition peak decreased linearly from $61.4{ }^{\circ} \mathrm{C}$ to $49.2^{\circ} \mathrm{C}$, which was comparatively larger than GLY. This result is also caused by the existence of isopropyl and propyl groups in PAN molecules which affect to the crystalline region in DSPC and set off lipid distortion. Although the results are not exact due to the existence of another phase occurred below $40 \%$ PAN, the overall variance of $\Delta \mathrm{H}$ showed almost linear-decreasing patterns as seen in Fig. 11(B) and the decreasing amount from $60.45 \mathrm{~J} / \mathrm{g}$ to $8.7 \mathrm{~J} / \mathrm{g}$ appeared to be similar to those of GLY. Over 55\% PAN, the thermotropic pattern was observed to be monotectic again and $\Delta \mathrm{H}$ also decreased linearly. Therefore, it can be concluded that PAN is well-swollen from $55 \%$ to $90 \%$.

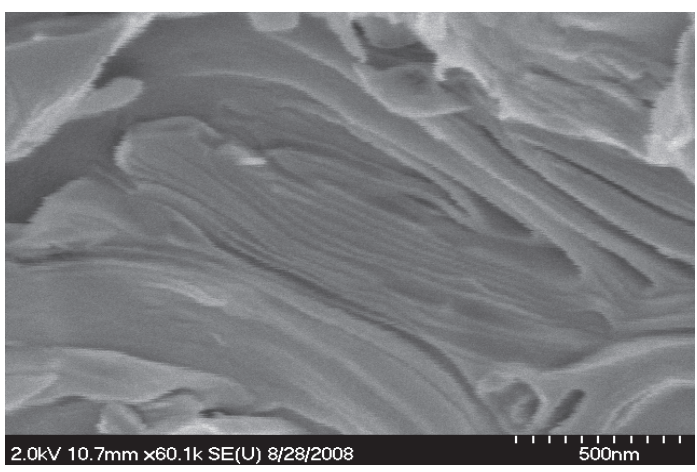

Figure 13. Cryo-SEM photographs of samples having the lamellar structure to be well-formed and well-swollen by PAN at $70.0 \% \mathrm{w} / \mathrm{w}$.

Cryogenic study for samples having the lamellar structure well-swollen by GLY and PAN with Cryo-SEM. Fig. 12 and 13 showed Cryo-SEM images of lamellar structures swollen by $70 \%$ of GLY and PAN, respectively.

All samples showed good images for well-formed lamellar structures as seen in Fig. 12 and Fig. 13. All samples were confirmed to be composed of multi-layers having several to over 10 layers. Therefore, it was possible to introduce GLY and PAN into DSPC lamellar phase instead of water.

\section{Conclusions}

In this study, we investigated the lyotropic mesomorphism of the lamellar structure in the case of incorporating glycerin and panthenol as a polar solvent which is not water, differently from many studies for lamellar structures formed in the mixed phase between lipids and water. Particulary, we investigated the transformation of DSPC lamellar structure dependent on the contents of polar solvents, Glycerin and Panthenol, and analyzed their swelling behaviors depending on relative hydrophilicities and lipophilicities between two solvents. Also, we confirmed the formation of those lamellar structures with Cryo-SEM. In those results, Glycerin was confirmed not to affect to the crystalline region in lamellar phase and be well-swollen in its non-crystalline region because of lacking lipophilicity. On the other hand, Panthenol was found to be well swollen in the non-crystalline region and slightly affect to the crystalline region due to the existence of isopropyl and propyl groups. In further study, lyotropic mesomorphism in the case of incorporating ceramides, cholesterol, and fatty acids which are composed of stratum corneum and act as a protective skin barrier will be examined to apply to alleviating atopy symptoms.

\section{References}

1. Elias P. M. J. Invest. Dermatol. 1983, 80, 44.

2. Potts, R. O.; Guy, R. H. Pharm. Res.1992, 9, 663.

3. Coderch, L.; De Pera, M.; Fonollosa, J.; De La Maza, A.; Parra, J. Contact Dermatitis 2002, 47, 139.

4. Nicolazzo, J. A.; Morgan, T. M.; Reed, B. L.; Finnin, B. C. J. Controlled Release 2005, 103, 577.

5. Lim, P. F. C.; Liu, X. Y.; Kang, L.; Ho, P. C. L.; Chan, Y. W.; Chan, S. Y. Int. J. Pharm. 2006, 311, 157.

6. Fang, J. Y.; Hwang, T. L.; Leu, Y. L. Int. J. Pharm. 2003, 250, 313. 
7. Rand, R. P.; Parsegian, V. A. Biochim. Biophys. Acta 1989, 988 , 351.

8. Binder, H.; Kohlstrunk, B.; Heerklotz, H. H. J. Colloid Interface Sci. 1999, 220, 235.

9. Markova, N.; Sparr, E.; Wadso, L.; Wennerstrom, H. J. Phys. Chem. 2000, 104, 8053.

10. Blumstein, A.; Blumstein, R. B.; Clough, S. B.; Hsu, E. C. Macromolecules 1975, 8, 73 .

11. Cevc, G.; Marsh D. Phospholipid Bilayers. Physical Principles and Models; Wiley: New York, 1987; p 442.

12. Marsh D. CRC Handbook of Lipid Bilayers; CRC Press, Inc.: Boca Raton, FL, p 387.

13. Zbytovska, J.; Kiselev, M. A.; Funari, S. S.; Garamus, V. M.; Wartewig, S.; Neubert, R. Chemistry and Physics of Lipids 2005, 138, 69.
14. Kodama, M.; Aoji, H.; Takahashi, H.; Hatta, I. Biochem. Biophys. Acta 1997, 1329, 61.

15. Wiener, M. C.; Suter, R. M.; Nagle, J. F. Biophys. J. 1989, 55, 315.

16. Wiener, M. C.; King, G. I.; White, S. H. Biophys. J. 1991, 60, 568.

17. Michael, S.; Jerry, M. Advanced organic chemistry : Reactions, Mechanisms, and Structure; John Wiley \& Son: 1992; p 21.

18. Jeong, T. H.; Oh, S. G. Bull. Korean Chem. Soc. 2007, $28,108$.

19. Klose, G.; Konig, B.; Meyer, H. W.; Schulze, G.; Degovics, G. Chemistry and Physics of Lipids 1998, 47, 225.

20. de Vringer, T.; Joosten, J. G. H.; Junginger, H. E. Colloid \& Polymer Science 1987, 265, 167.

21. Glatter, O.; Kratky, O. Small Angle X-Ray Scattering; Academic Press: New York, 1982.

22. Jimenez-Monreal, A. M.; Villalain, J.; Aranda, F. J.; Gomez-Fernandez, J. C. Biochimica et Biophysuca Acta 1998, 1373, 209. 